D0I: $10.33142 /$ ec. v2i3. 243

\title{
BIM技术在建筑工程施工管理中的应用探索
}

\author{
谭名燕 \\ 中国建筑第二工程局有限公司，重庆 400020
}

[摘要] BIM技术的应用已经体现出了一定的优势, 所以其现在成为了我国建筑工程领域当中应用较为广泛的技术。应用了BIM 技术后，其可以实现工程管理工作的可视化和高效化，对于工程质量的提高和工程进度的保证都可以起到重要作用，这就需要 广大建筑企业对这门技术给予足够的重视

[关键词]建筑施工管理; BIM技术; 应用

\section{Application of BIM Technology in Construction Management of Construction Engineering}

TAN Mingyan

China Construction Second Engineering Bureau Ltd., Chongqing, China 400020

\begin{abstract}
The application of BIM technology has shown some advantages, so it has become a more widely used technology in the field of building engineering in our country. With the application of BIM technology, it can realize the visualization and high efficiency of engineering management, and it can play an important role in the improvement of engineering quality and the guarantee of engineering progress. This requires the vast number of construction enterprises to give sufficient attention to this technology.
\end{abstract}

Keywords:Construction management; BIM technology; Application

引言

一个完整的建筑工程往往成本花费都十分的巨大, 并且会有大量的施工人员参与其中, 工程建造持续时间也会 较长。一个工程建造从开始到结束会历经多个阶段, 诸如: 前期的准备阶段, 工程建造阶段以及最后的交付使用阶 段。在建筑工程实际建造的时候, 工程建造的质量会受到各种不同因素的影响, 诸如：环境因素，气候因素以及诸 多的突发情况等等。除了外界环境的影响之外, 最为重要的是在工程建造中施工质量经常会受到人为操作的影响。 由于在工程建造之前需要安排专业人员实施前期的实地勘探以及设计工作, 进而要想在施工阶段确保工程结构的质 量务必要从前期准备工作入手来增强管理力度, 这样才可以更好的对工程的结构质量加以确保, 现如今在开展工程 施工管理工作的时候, 结合实际情况引用 BIM 技术能够有效的提升工作的效率, 从而对施工质量起到积极地影响。

\section{BIM技术概述}

BIM 其中文名称就是建筑信息化模型。就以往陈旧的建筑工程建造模式来说, 大多数都是利用 2D 或者是 3D 技术, 但是因为这些技术往往只可以对建筑结构形式加以仿照, 这种形式不能保证更加准确的获得工程结构的关键信息, 进而 $2 \mathrm{D}$ 以及 $3 \mathrm{D}$ 技术势必会在行业的发展中被淘汰, 在这个时候 BIM 技术应时而生。BIM 技术牵涉到的层面较多, 涉及到工程设计工作, 工程建造管理, 借助 BIM 技术可以综合所有的工程信息对建筑结构完成动态的模型的创建, 将 BIM 技术切实的运用到工程设计工作之中, 能够保证在较短的时间内来完成信息的高质量的传递, 为后续的施 工工作创造良好的基础 ${ }^{[1]}$ 。

\section{BIM技术的特点}

\section{1 可视化}

可视化可以结合获得的部件结构的信息来构建一个 $3 \mathrm{D}$ 的视觉效果。在建筑理论中，可视化技术拥有较强的实 际运用价值, 可以更好的发挥出良好的影响作用。现如今大量的工程信息通畅都是借助绘画来加以呈现的, 这种形 式通常需要操作人员对各个部件的结构进行简单的描述。但是在科学技术迅猛发展的带动下, 使得大量的高科技的 建造方式呗研发出来，BIM 技术的切实运用能够较好的解决传统建筑技术中存在的弊端 ${ }^{[2]}$ 。

\section{2 和解与谐和}

在工程实际建造中, 需要保证施工方与设计方能够进行通力合作。将 BIM 技术切实的加以运用可以更加高效的 
对工程中存在的各种问题加以确定，之后采用适当的方法来给予优化完善。

\section{3 动态模仿}

模仿其并非是单纯的对建筑结构的形式加以仿照, 将 BIM 技术运用到动态模仿工作之中, 可以有效的按照业主 的意愿来对建筑实施设计。结合高质量的模仿来为工程设计以及建造工作提供参考 ${ }^{[3]}$ 。

\section{4 更强的协调性}

将 BIM 技术切实的加以运用之后, 能够在设计部门与施工部门之间完成调节作用, 针对工程建造中遇到的各类 问题加以切实的解决, 借助 BIM 技术对施工遇到的问题实施综合分析研究, 联系专门的额模拟操作来对问题加以 有效的解决。但是单纯的借助二维设计图极易造成人们对碰撞的问题完全的忽视。

\section{5 可视化带来的模拟性}

模拟性其是 BIM 技术中十分突出的一个特性, 首先利用 BIM 技术的模拟性能够更加准确的对建筑结构的结构形 态进行模拟, 在将信息数据录入其中, 能够将内部构造进行还原。在工程正式建造之前, 务必要全面的收集数据信息, 利用这些信息对工程结构实施模拟，能够将完整的构造以 3D 图形的形式呈现出来。

\section{3 建筑施工管理中B IM技术的应用}

\section{1 设计阶段、组织阶段}

在针对施工图纸实施全面的深入的分析之后, 最为重要的是需要对原图纸实施优化, 设计需要与工程实际情况 相一致, 这样能够保证后期的工程建造工作按部就班的进行。在针对测绘实施切实的规划工作的时候, 需要充分 的结合现实情况开展各项工作, 施工人员务必要对施工设计实施全面的研究, 并且需要掌握施工机械的各项性能, 这样才能保证机械在施工中的作用更好的发挥出来。在施工中切实的引用 BIM 技术之后, 所有的编程信息可以在 $3 \mathrm{D}$ 模型中呈现出来, 这样能够有效地解决二位图形中存在的问题, 可以结合实际需求来进行适当的调整, 促使设 计能够更加的完美。借助 BIM 技术可以充分的联系现实状况来编制高效的施工计划, 并且依据施工计划来选择施 工技术 ${ }^{[4]}$ 。

\section{2 BIM在施工方案和工艺模拟中的应用}

借助 BIM 技术可以更加准确的创建三维图形, 之后为后续的项目设计、工程建造给与一定的指导。借助三维图 形能够制定出高质量的管理方案。BIM 技术能够对各种类型的工程结构济宁模拟, 这样对于工程施工中施工技术的 选择十分的有帮助。BIM 技术结合虚拟技术能够对工程建造中存在的各类重点和难点加以确定, 进而更够为工程施 工创造良好的基础 ${ }^{[5]}$ 。

\section{3 实现工程信息的集成化管理}

就当前的建筑行业来说, 信息量是十分巨大的, 操作人员的能力的不同也会使得信息数据分析结果的不尽相同, 这样就不能为后续的工作人员提供准确的信息。借助 BIM 技术能够有效地解决上述问题，所有的信息的分析都会 借助 BIM 技术来完成, 进而增强了信息分析结果的质量。这样就会地上的工作人员能够在较短的时间内获得需要 的细淢数据, 为工程建造周期的保证创造良好的基础。

\section{4 进度管理}

以往陈旧形式的工程管理模式都是借助进度图来开展各项工作的, 这种管理形式存在较多的弊端, 并且在解 决问题的时候具有一定的局限性。诸如, 如果管理人员不具备专业的资质, 就会对调度工作造成影响。如果将 BIM 技术引用到 5D 模型的构建工作之中, 工作人员可以结合实际情况来布置工程的各项流程, 之后将流程当做是网络 设计的借此狐, 促使网络规划能够构建完整的工程模型, 将时间维度的信息引入到模型之中, 这样能够有效地提升 模拟图的质量, 这样对于工程的施工能够创造良好的前提条件, 并且有利于管理工作按部就班的实施。

\section{5 成本管理}

关于建立项目, 成本投入较大, 并且工程的持续时长较长, 建立本钱由资料成本、设备成本、人工成本三个主 要组成局部组成。在项目管理的开展中, 有必要进一步细化本钱, 由于假如本钱构造过于复杂, 管理的有效性就会 降低。经过 BIM 管理, 管理能够变得愈加动态, 并且具有更高的及时性和精确性。确保成本管理的实践效果, 进 步资金核算的效率。BIM 管理基于计算机、通讯和其他技术，可以说具有较强的实用性和灵活性，并依据实践施工 条件树立成本管理数据库, 以确保有效处置资料、设备和劳动力等成本信息。在开端本钱管理时, 能够在数据库中 取得所需信息, 并在取得信息后运用计算机停止处置, 从而可以直接取得预算结果, 这样对于信息的准确性的保证 是非常有助益的。 


\section{结语}

目前建筑工程管理工作的开展会受到多个方面因素的影响, 所以工程管理质量的提高面临着很大的问题。BIM 技术的应用可能大大节约设计成本, 提高管理效率, 近年来其也得到了一定程度的推广, 其可以实现信息的实时共 享, 同时也可以及时发现施工中所存在的隐患。现在 BIM 技术体系还在为断完善, 在将来, 其也可以应用到施工、 设计、和运行维护工作等多个环节当中。

\section{[参考文献]}

[1] 李俊慷, 沈車, 张明. BIM技术在建筑工程施工管理中的应用解析 [J]. 工程建设与设计, $2019(02): 244-245$.

[2] 谢菪. BIM技术在建筑工程建设管理中的应用分析 [J]. 建材与装饰, 2018 (42): 184-185.

[3] 任国智. BIM技术在建筑工程施工管理中的应用探索 [J].智能建筑与智慧城市, 2018 (04)：67-68.

[4] 吴波. BIM技术在建筑工程施工管理中的应用探索 [J].中小企业管理与科技 (中旬刊), 2018 (02) : 145-146.

[5]王兴. BIM技术在建筑施工管理中的应用分析 [J].中国住宅设施, 2017 (08) : 111-112.

作者简介: 谭名燕, 男, (1991-05), 助理工程师, 从事建筑工程专业。 